\title{
Circuit
}

Musiques contemporaines

CIRCUIT

\section{American Composers Forum}

Se réseauter ou au-delà de la pige d'un soir dans le ghetto de la

nouvelle musique

American Composers Forum

Networking USA or Beyond the One-Night Stand in the New

Music Ghetto

\section{Philip Blackburn}

Volume 14, numéro 2, 2004

Montréal/Nouvelles Musiques

URI : https://id.erudit.org/iderudit/902315ar

DOI : https://doi.org/10.7202/902315ar

Aller au sommaire du numéro

Éditeur(s)

Les Presses de l'Université de Montréal

ISSN

1183-1693 (imprimé)

1488-9692 (numérique)

Découvrir la revue

Citer ce document

Blackburn, P. (2004). American Composers Forum : se réseauter ou au-delà de la pige d'un soir dans le ghetto de la nouvelle musique. Circuit, 14(2), 67-76.

https://doi.org/10.7202/902315ar

\section{Résumé de l'article}

Philip Blackburn dresse un portrait de l'American Composers Forum, organisation fondée en 1973 et qui a permis la création d'un réseau de diffusion de la nouvelle musique aux États-Unis. L'ACF compte 1700 membres et soutient des projets de création basés sur l'étroite collaboration entre les créateurs, les interprètes et la communauté. Il s'agit donc de soutenir la carrière des compositeurs en intégrant leurs oeuvres aux activités culturelles des communautés. Non seulement l'ACF permet aux oeuvres de circuler, mais elle assure leur création dans le cadre d'expériences musicales qui répondent aux besoins artistiques des communautés d'accueil. Il se crée alors un véritable marché de la nouvelle musique.
Tous droits réservés @ Les Presses de l’Université de Montréal, 2004

Ce document est protégé par la loi sur le droit d'auteur. L'utilisation des services d'Érudit (y compris la reproduction) est assujettie à sa politique d'utilisation que vous pouvez consulter en ligne.

https://apropos.erudit.org/fr/usagers/politique-dutilisation/ 


\section{American Composers Forum Se réseauter ou au-delà de la pige d'un soir dans le ghetto de la nouvelle musique}

Philip Blackburn

II y a quelques années, un organisme de nouvelle musique eut l'idée de faire circuler des partitions et des enregistrements dans différentes régions des États-Unis, de sorte que compositeurs et interprètes puissent prendre connaissance de ce qui se faisait ailleurs et programmer de nouvelles œuvres. Un circuit de dix lieux fut créé, chacun disposant du matériel pendant un mois. II fallut un certain temps pour organiser le tout et se rendre compte que les compositeurs inclus dans le projet se sentaient honorés. Un jour, la lourde malle arriva dans mon bureau. Nous avions invité des centaines de compositeurs et d'interprètes locaux à venir y jeter un coup d'œil. Personne ne vint. Un mois plus tard, nous l'avons envoyé à son escale suivante comme un chargement de déchets toxiques cherchant un endroit pour reposer en paix. Elle aurait eu plus de chance de trouver son auditoire si on l'avait envoyée à bord de la navette spatiale Voyager.

"Réseauter» (Networking) est notre principale raison d'être, mais le but ultime du travail que fait l'American Composers Forum (ACF) avec les créateurs, les interprètes et les collectivités n'est pas tant de réseauter que de donner aux artistes les outils dont ils ont besoin pour soutenir leur carrière et pour améliorer la situation à travers des expériences musicales. Aux États-Unis, nous devons construire non seulement des réseaux entre artistes pour favoriser de fructueuses collaborations, mais aussi des infrastructures pour le marché. "Nouveaux marchés pour la nouvelle musique » est notre cri de ralliement.

Avant que nous puissions développer un marché pour la nouvelle musique, nous devons d'abord faire savoir au public que tous les compositeurs ne sont pas morts (du moins pas encore). C'est ce que certains croient depuis que la musique a été éliminée de la plupart des écoles, et d'un peu partout d'ailleurs, sauf dans quelques téméraires stations de radio et magasins de disques.

Nous essayons d'abord et avant tout d'approfondir les liens personnels et locaux, de créer une communauté pour qui la nouvelle musique compte. Nous portons autant 
d'attention à intégrer les gens dans le processus créateur qu'à faire circuler un produit fini. La musique aux Etats-Unis n'étant plus un "sport de participation", plusieurs n'ont jamais fait l'expérience de jouer ou de chanter en groupe. La plus grande partie des pressions commerciales cherche à augmenter le nombre de consommateurs de culture. Qui souhaite un plus grand choix de musiques dont personne n'a voulues au départ?

Les commentaires qui suivent se fondent sur une décennie d'expérimentations au Forum et ne se veulent pas un survol de l'ensemble du monde des arts américains. Certaines idées paraîtront peutêtre hérétiques au regard des méthodes traditionnelles de soutien aux arts, mais elles reflètent les besoins comme nous les percevons et la manière dont nous avons su les combler avec un certain succès en nous appuyant sur le fait suivant : nous sommes toujours là alors que plusieurs ont dû fermer leurs portes.

\section{Mission}

L'American Composers Forum établit des liens entre les collectivités, les compositeurs et les interprètes, encourageant la création, I'interprétation et l'appréciation de la nouvelle musique.

En construisant des relations entre artistes et publics, le Forum développe des programmes destinés à l'éducation des auditoires d'aujourd'hui et de demain, à dynamiser les carrières des compositeurs et des interprètes, à stimuler l'esprit d'entreprise et la collaboration, à favoriser la créativité musicale et à servir de modèles de soutien efficace aux arts.

\section{Carte de la distribution des membres}

Nous ne sommes qu'un organisme parmi une poignée de services aux compositeurs aux États-Unis; parmi les autres, on retrouve l'American Music Centre, Meet the Composer et la Society of Composers. Chacun joue un rôle complémentaire sur l'échiquier national, quoique l'on fasse peu de chose sur une base quotidienne pour coordonner les activités des uns et des autres. 


\section{Statistiques}

Fondé en 1973

Le siège social n'est pas à New York et, malgré tout, c'est un organisme national!

1700 membres

14 employés +10 sections régionales

Budget annuel : 2,9 millions dont :

$75 \%$ en dons personnels ou de fondations privées

$16 \%$ en subventions gouvernementales

$7 \%$ en revenus de ventes

$2 \%$ en adhésion

L'ACF est un organisme à but non lucratif qui dépend des fondations et de leurs priorités. Sa tâche est d'équilibrer les besoins et les désirs des artistes avec les aspects pratiques de la levée de fonds. Dans plusieurs cas, il se retrouve à faire l'éducation non seulement des compositeurs (dont la mentalité est relativement individualiste) et des collectivités (qui utilisent la musique mais ne pensent jamais à rétribuer les compositeurs), mais aussi des donateurs (qui ont leurs propres raisons de donner de l'argent et ne sont souvent pas très chauds à l'idée de prendre des risques).

\section{Défis}

- Déclin de l'intérêt des donateurs face à "l'art pour l'art»: "les concerts de musiques nouvelles sont morts".

- Déclin de l'économie et diminution de l'enveloppe globale des dons : il en résulte un marché du don plus compétitif.

- Hausse des attentes des donateurs envers de substantielles évaluations de projets.

- Pression sur les organisations artistiques pour fournir des composantes éducatives en parallèle avec leurs programmes réguliers.

- Indigence des donateurs dans les banlieves ef les régions rurales, alors que la demande pour des programmes artistiques dans ces milieux augmentent.

- Incapacité de garantir qualité et attrait des œuvres de nouvelle musique. 


\section{Possibilités}

1. Un bassin national de compositeurs talentueux qui écrivent dans une large variété de styles et conçoivent des activités créatives.

2. Une ouverture de plus en plus grande aux compositeurs et à leurs œuvres.

3. Un intérêt croissant des donateurs pour le rôle des arts dans le développement des collectivités.

4. Un intérêt croissant envers la commande d'œuvres musicales pour célébrer des événements spéciaux.

5. Demande de ressources et de matériel didactique pour l'éducation musicale.

6. Évolution de technologies qui peuvent fournir contenus et produits à un auditoire de plus en plus large.

Le Forum s'emploie à soutenir la croissance artistique des compositeurs et à développer de nouveaux marchés pour leur musique.

En voici les principaux éléments.

\section{Les sections régionales}

Les dix sections régionales du Forum fournissent les infrastructures nécessaires pour lier les collectivités aux compositeurs et aux interprètes, aux administrateurs des programmes nationaux du Forum et aux programmes incitatifs répondant localement aux besoins spécifiques des interprètes et des collectivités.

Par cette présence locale, l'organisation engage les artistes et les collectivités de manière plus directe, vivante et efficace. Leur constante présence permet de forger des alliances durables avec un vaste éventail de partenaires musicaux, culturels, éducatifs et philanthropiques.

Les dix bureaux régionaux sont situés à Atlanta, Boston, Chicago, Los Angeles, Twin Cities, Greater Minnesota, New York City, Philadephie, San Francisco et Washington D. C.

Si les artistes forment le niveau cellulaire, les sections régionales représentent le niveau "petit organisme" de l'organisation. Elles opèrent sur le terrain et maintiennent une présence locale forte, non en présentant des concerts, mais en travaillant à intégrer les compositeurs aux activités des écoles, des églises, des musées scientifiques, des maisons pour femmes battues, des groupes de voisins, des programmes jeunesse de réinsertion sociale.

En étant sur place, elles peuvent aider les membres à mieux définir leur identité, à créer des chants, des danses, des vidéos, du théâtre, des mixages électroniques, 
des installations sonores, ou tout ce qui peut être demandé. Nous constatons souvent que plus une œuvre s'éloigne de la musique de la communauté, plus la présence du compositeur est appréciée.

Les sections régionales tiennent fréquemment des salons, organisent des rencontrent avec des ensembles locaux et des séminaires sur les aspects techniques de la poursuite d'une carrière en arts. Elles organisent des rencontres avec des artistes d'autres disciplines et offrent un cercle social immédiat aux artistes qui s'installent dans leur région. Les sections régionales ont leur propre directeur local à temps partiel. Le développement du système des sections régionales fut au départ subventionné par une fondation, mais nous sommes en train de le transformer selon le modèle des franchises, dans lequel les sections régionales, subventionnées localement, sont des branches affiliées indépendantes de l'organisation nationale.

Sounding Board, le bulletin de liaison mensuel, est un outil de réseautage pour toutes les constituantes du Forum. II donne des nouvelles des derniers programmes, des changements artistiques et communautaires qu'ils ont pu engendrer, des possibilités qui s'offrent à travers le monde lappels d'offres pour de nouvelles partitions, concours, etc.) et propose des rencontres compositeurs-interprètes.

\section{Les prix}

Le Composers Commissioning Program et les McKnight Fellowships fournissent des sommes substantielles aux compositeurs tout en stimulant les collaborations et les résidences.

Plusieurs programmes nationaux de commandites d'œuvres ont disparu au cours des dernières années, faisant du Composers Commissioning Program un des derniers survivants. Il est ouvert aux compositeurs de la relève qui font une inscription conjointe avec un interprète. Toutefois, l'un des deux doit être de la ville de New York ou de l'État du Minnesota (pour satisfaire aux exigences de la fondation qui le subventionne). Ces restrictions géographiques, frustrantes pour certains, servent en fait à stimuler le commerce et le réseautage «inter-États». En effet, plusieurs relations nouvelles s'établissent dans les semaines précédant la date limite d'inscription, alors que certains ensembles et compositeurs cherchent encore un partenaire. II résulte de tout cela que les ensembles du Minnesota qui, il y a 20 ans, inscrivaient rarement de la nouvelle musique à leur programme, le font maintenant régulièrement.

Les McKnight Fellowships sont des prix de distinction attribués aux compositeurs du Minnesota. Ils comportent un fonds sans restriction (de $25000 \$$ par an) et une bourse de projet optionnel pour laquelle chaque compositeur peut proposer son propre concept de résidence communautaire et recevoir une somme additionnelle de $5000 \$$ pour le réaliser. Tous les compositeurs ne sont pas faits pour le travail communautaire, mais plusieurs s'y sont convertis pour bénéficier de ce programme ou d'un autre. Cet 
aspect du programme n'est pas une commandite; on demande plutôt au compositeur de stimuler le travail créateur chez d'autres. Certains projets ont eu lieu dans des réserves, d'autres dans des synagogues ou encore dans des studios de télévision.

Certains préjugés voudraient que le fait d'écrire pour une collectivité autre qu'un ensemble de nouvelle musique suppose des compromis ou une simplification stylistique de la part des compositeurs. Ceux-ci seraient obligés de faire du travail social dans des soupes populaires. Mais les compositeurs sont souvent surpris du degré de raffinement de leurs partenaires et de leur réception enthousiaste. De même, on craint que les gens soient incapables de savoir ce qu'est une musique de qualité ou de sélectionner un bon compositeur pour travailler avec eux. L'expérience nous a appris que ce n'est pas vrai.

\section{L'aide à l'interprétation}

Subito, Moving Music et le Encore Fund fournissent des outils pour des projets de fond. Sonic Circuits Electronic Music Festival fait la promotion d'œuvres de nouveaux venus.

\section{Programme Subito}

Notre responsabilité nous oblige à nous adapter aux demandes changeantes du milieu. Un gros programme de commandites n'est pas pertinent si tout ce dont une personne a besoin, c'est d'un ingénieur du son pour un spectacle la semaine suivante ou d'une gardienne pendant la générale. Les artistes sont économes et souvent un petit montant peut leur faire faire un bon bout de chemin vers une réalisation significative.

Subito répond à ces besoins. II offre un service rapide de bourse, sans date limite ni jury (remplacé par une simple évaluation de pairs), fournit une réponse rapide et n'est pas normatif. "Que voulez-vous faire? " "Comment 500 \$ à 1500 \$ peuvent donner un coup de pouce à votre carrière?"

Cette structure, en comparaison de processus plus normatifs de bourses qui laissent de côté plusieurs projets valables, permet au programme de combler des besoins spontanés, encourage le risque artistique et renforce le développement professionnel. Elle respecte l'autodétermination des artistes et ne les force pas à s'ajuster au moule des arcanes bureaucratiques et de se retrouver à faire un projet autre que celui qui leur tenait à cœur. Si ce programme est des plus inquiétants pour les donateurs, il s'avère des plus efficaces sur le plan financier, comme sur les plans de la rapidité des résultats et de la satisfaction du consommateur.

\section{Programme Moving Music}

Moving Music remplit la même fonction à l'égard des chorégraphes qui ont besoin d'aide lorsque vient le moment d'annexer de la nouvelle musique à leurs danses. Les chorégraphes comptent parmi les plus importants utilisateurs de musiques nouvelles et, pourtant, le manque de fonds les conduit souvent à prendre des raccourcis, parfois 
illégaux, avec la musique, comme de ne pas payer les droits d'auteur aux compositeurs. Une petite carotte est souvent tout ce dont ils ont besoin pour réaliser leurs rêves de travailler avec des musiciens sur scène, ce qui est un exemple de réseautage entre différents genres.

\section{Programme Encore Fund}

Encore Fund offre de petites bourses aux ensembles qui désirent jouer des œuvres déjà créées qu'ils n'ont jamais mises à leur programme. Ils s'engagent à les jouer au moins trois fois en dix-huit mois. Ce fonds aide les compositeurs à voir leurs œuvres reprises, parfois s'ajouter au répertoire d'un groupe ; il favorise ainsi la rencontre entre compositeurs et interprètes.

Cet outil de réseautage est facile à gérer et très efficace, car il résulte en dizaines d'interprétations pour environ le coût d'un seul concert. Ce fonds encourage les ensembles classiques à prendre plus de risques à l'égard de la musique contemporaine, ce qui élargit du coup l'auditoire potentiel du compositeur.

\section{Sonic Circuits}

Sonic Circuits' invite tous ceux qui font de la "musique qui demande de brancher quelque chose" de nous la soumettre. Nous conservons une liste d'environ 30 pièces dans quatre catégories : bande magnétique/CD seul, vidéo d'art, pièce qui peut être jouée à partir d'une partition (ex. piano et bande magnétique) et actions vivantes où les gens interprètent leur propre musique. Cela se présente comme une trousse de programmation "faites-le-vous-même", offerte gratuitement à quiconque voudrait l'examiner en vue de l'inclure dans sa programmation. Nous avons jusqu'à 90 lieux intéressés par ce service. Plusieurs d'entre eux juxtaposent leur propre travail local ou celui d'étudiants à celui d'artistes internationaux. Certains ont des budgets pour engager des artistes sur scènes mais les événements n'ont pas nécessairement à respecter le format traditionnel du concert. II peut s'agir d'installations dans des galeries d'art, d'émissions de radio, d'intermissions au théâtre. Les cours extérieures ont toutes été utilisées pour ce genre d'événements.

Sonic Circuits sert efficacement à créer de larges intersections entre artistes et publics, puisqu'il est peu coûteux à faire fonctionner et qu'il mêle l'électroacoustique institutionnalisée ef l'électronique de club. Un plus grand nombre de subventions aux interprètes permettrait d'élargir le volet tournée du festival, tout en préservant le caractère local demandé par le commanditaire.

\section{Les médias}

Chaque jour, par l'intermédiaire du Composers Datebook, de innova Recordings et de nombreux sites Internet, le Forum se sert des médias pour relier les compositeurs au public. 


\section{Composersdatebook.org}

Le Forum n'a pas pour objet de présenter des concerts de nouvelle musique, contrairement à ce que certaines personnes pourraient croire. Nous pensons qu'un changement sociétal plus profond doit se produire avant que les concerts, peu fréquentés, puissent être un moyen viable pour les compositeurs de diffuser leur musique. Pour parvenir à cette fin, nous produisons quotidiennement une émission sur un réseau de 250 stations de radio publique. Composers Datebook est une capsule de deux minutes qui s'adresse aux mélomanes (et animateurs de radio conservateurs). Elle juxtapose "aujourd'hui dans I'histoire de la musique classique" et une œuvre récente de même facture, pour rappeler aux auditeurs que toute musique fut un jour nouvelle.

\section{Innova.mu}

Innova Recordings, la compagnie de disque du Forum, produit chaque année 25 disques compacts de musique nouvelle "classique», jazz, expérimentale, électronique et "world". II s'agit d'un organisme à but non lucratif qui opère en partie sur le modèle d'un service, en partie sur celui du monde des affaires. Ce mode de fonctionnement veut dire que nous voyons à la fabrication, au marketing et à la distribution de nouveaux enregistrements, alors que l'artiste est responsable des coûts de production (ils peuvent être couverts par notre programme de prêts) et conserve tous les droits et $100 \%$ des ventes. Les disques compacts sont la monnaie de la nouvelle musique, un moyen pour les artistes de faire des gains financiers ou du moins d'obtenir une plus grande visibilité. Comme l'entreprise ne dépend pas des ventes (nous facturons un montant minimal en plus du coût de production), nous pouvons prendre de plus grands risques artistiques contrairement à la plupart des autres compagnies, et ne pas fonder nos décisions sur le succès ou l'échec commercial.

Tous les titres innova sont diffusés sur l'une de nos cinq stations de radio Internet, ce qui rend les plus récentes productions accessibles à partir d'un ordinateur. Nous avons aussi une série de titres imprimables sur demande à partir de notre site Internet seulement, ce qui diminue encore plus les risques financiers pour les artistes.

\section{Les programmes éducatifs}

BandQuest, Composers Suitcase et des compositeurs en résidence dans les différentes sections régionales du Forum instruisent la prochaine génération de musiciens et d'auditeurs, tout en permettant aux compositeurs d'évoluer dans un cadre éducatif.

\section{Banquest.org}

Les compositeurs font souvent de la ségrégation entre eux, s'étiquetant comme compositeurs de groupe, de chœur, de danse ou de carillon. Nous croyons à l'avantage 
de faire sauter les barrières. Le répertoire des harmonies scolaires est suffisant pour tuer dans l'œuf toute passion chez les jeunes musiciens (qui s'ennuient lorsqu'ils sont laissés à eux-mêmes). Nous avons commandé une anthologie de 12 courtes pièces de compositeurs de différents styles. Chacune se rapporte à un élément du programme scolaire (par exemple, I'histoire chinoise, les statistiques ou l'esclavage) abordé dans un cédérom, qui accompagne la partition.

Composers Suitcase ramène la créativité musicale à l'école primaire, d'où elle a été bannie par les coupures budgétaires. Une grille de normes d'apprentissage (Goals 2000) a été établie par le gouvernement, mais sans professeurs de musique pour l'implanter. Composers Suitcase contient des activités musicales, des exercices, des instruments et des projets qui peuvent être utilisés par n'importe quel professeur, même s'il n'est pas spécialisé en musique.

\section{Les résidences en communauté}

Des projets comme le Continental Harmony, le Faith Partners et les Community Partners des sections régionales offrent aux compositeurs la chance de travailler avec diverses collectivités pour faire connaître leur musique à de nouveaux auditoires. II en est de même dans le cas des programmes Museums, Composers, and Communities et McKnight Visiting Composer.

Faith Partners remet les compositeurs à la place qu'ils ont occupée historiquement pendant des siècles, c'està-dire dans les églises et les synagogues... mais avec une variante. Nous établissons un regroupement de trois lieux de culte différents; ils définissent ensemble la musique qu'ils voudraient avoir pendant l'année suivante. Nous lançons un appel auprès des compositeurs, et le candidat retenu écrit d'abord trois pièces, destinées à voyager d'un lieu à l'autre, puis trois autres, à l'intention de chaque communauté spécifique. Cela crée des liens interconfessionnels ou œcuméniques qui offrent des défis culturels et musicaux au compositeur, ainsi qu'un auditoire captif et particulièrement attentif chaque semaine. Plus que tout autre, ce programme a amené les compositeurs à se percevoir comme des travailleurs artistiques apportant leur contribution à la vie d'une communauté.

Deux artistes "en résidence» sont invités chaque année à venir réaliser au Minnesota un projet de leur choix, dans le cadre du programme McKnight Visiting Composer. Nous avons constaté que les plus efficaces de ces résidences sont celles qui durent au moins deux mois et qui se tiennent en marge des institutions et des circuits musicaux courants. Voici quelques exemples récents : une performance organisée par Fred Ho avec les membres de l'Hotel and Restaurant Workers Union : il y avait 17 langues différentes et plusieurs des travailleurs ont partagé pour la première fois leur culture avec celle de leurs collègues; Kenny Endo a dirigé un atelier de percussions Taiko dans un circuit d'écoles; Janek Schafer a créé à une 
installation sonore avec des ballons et des informations météorologiques; Dan Senn a nettoyé une vieille grotte chargée d'histoire locale et créé une installation vidéo (1 500 fermiers ont traversé des champs de bove pour venir la voirl.

Museums, Composers, and Communities met un compositeur en relation avec le Museum Loan Network (réseau de prêts). L'organisme met sur pied des expositions itinérantes d'œuvres d'art pour lesquelles un compositeur crée une œuvre musicale ou un environnement sonore.

Le documentaire Continental Harmony, d'une durée d'une heure et produit par PBS-TV, a mené notre philosophie sur la scène nationale. Sa diffusion régulière lui permet de continuer à susciter beaucoup d'intérêt. II montre comment nous avons semé des graines de nouvelle musique dans le sol de la nation, afin de rendre les compositeurs partie prenante essentielle du tissu social et de signifier que la vie sans eux est impensable. 